\title{
Clinical utility of alpha fetoprotein and HCCR-1, alone or in combination, in patients with chronic hepatitis, liver cirrhosis and hepatocellular carcinoma
}

\author{
Peng Jirun ${ }^{\mathrm{a}}$, Guoxin Zhang ${ }^{\mathrm{b}}$, Hyun Kee Kim ${ }^{\mathrm{c}}$, Seon-Ah Ha ${ }^{\mathrm{c}}$, Jin Zhongtian ${ }^{\mathrm{a}}$, Qiao Shishi ${ }^{\mathrm{a}}$, \\ Cui Zhuqingqing ${ }^{\mathrm{a}}$, Gong Lei ${ }^{\mathrm{a}}$, Jinah Yoo $^{\mathrm{c}}$, Sanghee Kim ${ }^{\mathrm{c}}$, Yong Gyu Park ${ }^{\mathrm{e}}$, Jing Wang ${ }^{\mathrm{b}}$, Yang Yang ${ }^{\mathrm{b}}$, \\ Zekuan Xu ${ }^{\mathrm{b}}$, Zuhu Huang ${ }^{\mathrm{b}}$, Yun Kyung Lee ${ }^{\mathrm{c}}$, Eun Young Song ${ }^{\mathrm{f}}$ and Jin Woo Kim ${ }^{\mathrm{c}, \mathrm{d}, *}$ \\ ${ }^{a}$ Department of Surgery, Peking University People's Hospital, Beijing, P. R. China \\ ${ }^{\mathrm{b}}$ Department of Gastroenterology, The first affiliated hospital of Nanjing Medical University, Nanjing, P. R. China \\ ${ }^{\mathrm{C}}$ Molecular Genetic Laboratory, College of Medicine, The Catholic University of Korea, Seoul 137-040, Korea \\ ${ }^{\mathrm{d}}$ Departments of Obstetrics and Gynecology, College of Medicine, The Catholic University of Korea, Seoul \\ 137-040, Korea \\ ${ }^{\mathrm{e}}$ Departments of Biostatistics, College of Medicine, The Catholic University of Korea, Seoul 137-040, Korea \\ ${ }^{\mathrm{f}}$ The Korea Research Institute of Bioscience and Biotechnology, Daejeon, Korea
}

\begin{abstract}
Serum alpha fetoprotein (AFP) is the most widely used tumor marker in detecting patients with hepatocellular carcinoma (HCC). However, it has been indicated that HCCR-1 (human cervical cancer oncogene 1) might be supplementary to AFP in the detection. We conducted a prospective study in 120 normal and 524 liver disease patients to evaluate the significance of simultaneous measurement of 2 tumor markers (AFP and HCCR-1) in the diagnosis of HCC through the cohort study in Korea and China. We also performed immunohistochemical studies using 25 normal subjects (N), 32 liver cirrhosis (LC) and 116 HCC tissues. The sensitivities of AFP (20 ng/mL) and HCCR-1 (10 ng/mL) in HCC were 55.8\% (164/294) and 44.2\% (130/294), respectively. When AFP was combined with HCCR-1, sensitivities increased to $4.2 \%(\mathrm{~N}), 12.7 \%$ (chronic hepatitis; $\mathrm{CH}$ ), $50.0 \%$ (LC), and $77.2 \%$ (HCC), respectively. Although there was no significant difference in the diagnostic rate for HCC between AFP and HCCR-1, many cases for AFP-negative HCC were positive for HCCR-1 and vice versa. Moreover, the combined use of AFP and HCCR-1 improved the diagnostic rate to $70.8 \%$ in small $\mathrm{HCC}(<2 \mathrm{~cm})$ and $81.6 \%$ in large $\mathrm{HCC}(\geqslant 2 \mathrm{~cm})$, respectively. AFP and HCCR-1 are independent markers. Our result suggests that the HCCR-1 could be an useful biomarker for HCC while the diagnostic rate could be significantly improved in the combined use of HCCR-1 and AFP.
\end{abstract}

Keywords: HCC, biomarker, AFP, HCCR-1

\section{Abbreviations}

HCC, hepatocellular carcinoma;

$\mathrm{CH}$, chronic hepatitis;

LC, liver cirrhosis;

HCCR-1, human cervical cancer oncogene 1;
AFP, alpha fetoprotein;

AFP-L3, Lens culinaris agglutinin-reactive AFP;

PIVKA-II, protein induced by vitamin $\mathrm{K}$ absence or antagonist-II;

DCP, des- $\gamma$-carboxy prothrombin.
* Corresponding author: Prof. Jin Woo Kim, Molecular Genetic Laboratory, College of Medicine, The Catholic University of Korea, Seoul 137-040, Korea. Tel.: +82 119014 2389; Fax: +82 2593 2389; E-mail: jinwoo@catholic.ac.kr.

\section{Introduction}

Hepatocellular carcinoma (HCC) is one of the most frequent malignant tumors and is the second and third 
most common causes of cancer death in China and Korea, respectively [1-3]. Being the fifth most common cancer in the world, HCC remains an important disease [4]. It is responsible for around 250,000 deaths every year [5]. Therefore, it is very important to detect this disease and the recurrence at its earlier period [6].

Currently, the only available tools for HCC surveillance are testing for serum AFP level and hepatic ultrasonography [7,8]. However, AFP level has poor sensitivity and specificity for HCC [7] and ultrasonography is operator dependent and limited in its ability to discriminate HCC from such non-neoplastic lesions as regenerative nodules [8]. Newer methods for the early detection of HCC are needed in the form of either biomarkers or radiological tests. The marker should be easily measurable, and the test should be reproducible, minimally invasive, and acceptable to patients and physicians [9].

Although serum AFP level is an useful tumor marker for the detection and monitoring of HCC, the falsenegative rate with AFP level alone may be as high as $40 \%$ for patients with early stage HCC. This means there is a possibility that the new HCC may be of the non-AFP secreting variety. Even in patients with advanced $\mathrm{HCC}$, the AFP levels may remain normal in $15 \%-30 \%$ of the patients. Other HCC markers, such as DCP, alkaline phosphatase isoenzyme-I, and tissue polypeptide specific antigen, have been developed to improve the sensitivity, specificity, early detection, and prediction of prognosis; but the overall results have been unsatisfactory $[10,11]$. In recent years, clinically useful tumor markers for HCC diagnosis have included AFP, a fucosylated variant of the AFP glycoprotein which has a high affinity to the sugar chain of Lens culinaris (AFP-L3), and DCP. Among them, AFP and AFP-L3 tests were approved by the Food and Drug Administration (FDA) and the DCP test is currently under review. These markers will definitely be helpful in evaluating and managing patients with $\mathrm{HCC}$ or those at risk for HCC in medical practice.

Recently several serum biomarkers such as glypican3 [12], alpha-1-acid glycoprotein (AAG) [13], and Cystatin [14] were also introduced to detect HCC. For example, GPC3 protein is a sensitive and specific serum marker for diagnosis of early HCC. Its expression in liver tissues can be used to discriminate tumor cells from benign hepatic cells [12]. The combination of AAG and AFP also showed higher sensitivity and improved the accuracy of HCC diagnosis [13]. SELDI-TOF MS also accurately distinguishes patients with HCC from those with hepatitis $\mathrm{C}$ virus cirrhosis, and is more ac- curate than traditional biomarkers in identifying small tumors [14].

Several laboratories have recently reported that HCCR-1 is expressed by a large proportion of HCCs, but is undetectable in normal hepatocytes and nonmalignant liver disease [3,15-18]. Elevated level of HCCR-1 has been shown to be associated with an increase in the risk of developing HCC [15]. We identified a new oncogene associated with human cervical cancer that is also overexpressed in various human tumors including liver cancer $[15,19,20]$. The HCCR (human cervical cancer proto-oncogene) is classified into two splicing isoforms, HCCR-1 (GenBank Accession number AF 195651) and HCCR-2 (GenBank Accession number AF 315598) [19]. Previous work suggests that cells expressing either HCCR-1 or HCCR-2 are tumorigenic in nude mice [19]. Northern and Western blot analyses and immunohistochemical studies indicate that the HCCR-1 mRNA and protein were overexpressed in tumorous compared with non-tumorous cirrhotic tissue of HCC [15]. These results indicate that $H C C R-1$ probably represents a new oncogene that is related to liver tumorigenesis, functioning as a negative regulator of the p53 tumor suppressor $[19,20]$. Moreover, the expression of HCCR-1 is modulated by the phosphatidylinositol 3-kinase/Akt pathway which is involved in the tumorigenesis by regulating cell cycle progression and survival [21].

Although we had previously developed HCCR-1 indirect ELISA assay for serological test [12], the development of sandwich ELISA assay was essential to resolve the specificity issue. To achieve this goal, we developed HCCR-1 sandwich ELISA assay in this study to improve the serological assay using HCCR-1 for LC or HCC. The HCCR-1 sandwich ELISA assay revealed that elevated HCCR-1 levels are associated with an increase in the risk of developing LC or HCC.

In this study, we undertook the following aims: 1) To investigate the diagnostic efficiency of HCCR-1 in LC or HCC, 2) To compare HCCR-1 with AFP test, 3) To investigate its usefulness in the early diagnosis for small HCC $(<2 \mathrm{~cm}), 4)$ To test for the combined analysis of HCC with AFP and HCCR-1 especially in small HCCs.

\section{Materials and methods}

\subsection{Patients and study design}

120 normal healthy subjects and 524 liver disease patients were recruited during the study period from 
September 2007 to December 2008 from 2 clinical sites (430 from Catholic University, Korea and 214 from Peking University, China). The patient cohort consisted of 393 males $(75.0 \%)$ with an average age of 54.7 years and 131 females $(25.0 \%)$ with an average age of 56.9 years. All blood samples for HCCR-1 were frozen for analysis and shipped to our institution. Clinical data were collected for serum chemistries, imaging (CT, MRI and/or ultrasound), presenting symptoms, medications/interventions/therapeutics, and other medical informations including the presence of hepatitis. Two tumor markers for HCC, AFP and HCCR-1, were measured in the serum samples obtained from 120 normal healthy volunteers $(\mathrm{N}), 110$ chronic hepatitis $(\mathrm{CH}), 120$ liver cirrhosis (LC) and 294 HCC patients.

Subjects were those with newly diagnosed HCC at the study onset. The preoperative diagnosis of HCC based on the diagnostic criteria for HCC used by the European Association for the Study of the Liver (EASL) [7]. HCC was diagnosed by at least two radiological imaging showing characteristic features of $\mathrm{HCC}$; or one radiological imaging showing characteristic features of HCC associated with AFP > $200 \mathrm{ng} / \mathrm{ml}$; or cytologic/histologic evidence. The staging of HCC was according to the criteria of American Joint Committee on Cancer (AJCC)/International Union Against Cancer (UICC) Tumor-Node-Metastasis (TNM) staging for liver tumors (sixth edition, 2002) [22,23]. None of the patients had other malignancies or active pulmonary disease. All patients and controls were subjected to the analysis with individual consent for the study. The use of blood samples was approved by the ethics committee of each 2 institution.

\subsection{Immunohistochemistry}

Immunohistochemical studies were performed using 25 paracancerous liver tissues, 18 decompensating LC, 14 compensating LC and 116 HCC tissues. Polyclonal antibodies directed against amino acids 167-360 of HCCR-1 were generated [15]. By using polyclonal antibodies, the immunostaining was performed at room temperature by using the UltraSensitive SP immunohistochemistry kit (Maixin Biotech, Fuzhou, China). PBS replaced the murine polyclonal anti-HCCR-1 serum (1:100) as a negative control. Using the Allred 8-unit system, we determined the tumor epithelial cell proportion score and intensity score. The proportion score included the fraction of positively stained tumor cells and was as follows: $0=$ none, $1 \leqslant 1 / 100$ th; $2=$ $1 / 100$ th to $1 / 10$ th; $3=1 / 10$ th to $1 / 3 ; 4=1 / 3$ to $2 / 3 ; 5$ $\leqslant 2 / 3$. The staining intensity score was as follows: 0 $=$ none; 1 = weak; 2 = intermediate; $3=$ strong. For the statistical analysis, an HCCR-1 score of 1-3 was considered low, an HCCR-1 score of 4-6 was considered intermediate, and an HCCR-1 score of 7-8 was considered high.

\subsection{Detection of HCCR-1 in serum by sandwich ELISA}

ELISA used in this study is an enzymatically amplified "two-step" sandwich-type immunoassay. The HCCR-1 levels were measured using a sandwich ELISA. The standard curve was constructed with serial dilutions of HCCR-1 $167-360$, ranging from 20 to $320 \mathrm{ng} / \mathrm{ml}$. By using 20, 40, 80, 160, and $320 \mathrm{ng} / \mathrm{ml}$ of HCCR-1 $167-360$ protein, a calibration curve was prepared.

In the assay, standards, controls and unknown serum samples are incubated in microtitration wells which have been coated with anti-HCCR-1 monoclonal antibody $(\mathrm{mAb}) 54115$. After incubation and washing, the wells are treated with another anti-HCCR-1 detection polyclonal antibody labeled with the enzyme horseradish peroxidase (HRP). After a second incubation and washing step, the wells are incubated with the substrate tetramethylbenzidine (TMB). An acidic stopping solution is then added and the degree of enzymatic turnover of the substrate is determined by dual wavelength absorbance measurement at 450 and $620 \mathrm{~nm}$. The absorbance measured is directly proportional to the concentration of HCCR-1 present in serum. A set of HCCR-1 standards is used to plot a standard curve of absorbance versus HCCR-1 concentration from which the HCCR-1 concentrations in the unknowns can be calculated. The reference ranges were obtained by receiver operating characteristics (ROC) curve analyses: $10 \mathrm{ng} / \mathrm{ml}$ was used as a cutoff level for HCC.

\subsection{Serum HCCR-1 and AFP concentrations}

The cut-off value to determine the positive rate was $10 \mathrm{ng} / \mathrm{ml}$ for HCCR-1 derived from generating ROC curve using 880 patients of HCC and 380 patients of $\mathrm{LC}$ or $\mathrm{CH}$ as a control. We then analyzed the test cohort consisting of 120 normal subjects, 110 chronic hepatitis patients, 120 liver cirrhosis patients, and 294 HCC patients to determine the positive response.

Serum levels of HCCR-1 were presented as the mean $\pm \mathrm{SD}$ and compared using Wilcoxon rank sum test and Kruskal-Wallis test. The AFP level was measured 
Table 1

HCCR-1 expression in HCC, LC and paracancerous tissues

\begin{tabular}{lrrcrlr}
\hline & Case & \multicolumn{2}{c}{ HCCR-1 } & $P^{\prime}$ & $P$ \\
\cline { 3 - 5 } & & positive & negative & & \\
\hline HCC & 116 & $94(81.03 \%)$ & $22(18.97 \%)$ & & \\
LC $\quad$ decompensating & 18 & $12(66.67 \%)$ & $6(33.33 \%)$ & 0.210 & $0.011^{\star}$ \\
& compensating & 14 & $7(50 \%)$ & $7(50 \%)$ & $0.015^{\star}$ & \\
Paracancerous tissues & 25 & $11(44 \%)$ & $14(56 \%)$ & $<0.001$ & $<0.001$ \\
\hline
\end{tabular}

${ }^{\star}$ Fisher's exact test.

$P^{\prime}$ : Comparing paracancerous tissues, compensationg cirrhosis, and decompensating cirrhosis with HCC.

$P$ : Comparing paracanerous tissues, combined compensating cirrhosis and decompensating cirrhosis with HCC.

by an enzyme immunoassay (EIA) (Elecsys Modular Analytics E170, Roche Diagnostics, Mannheim, Germany). To determine positive responses, we used the value of $20 \mathrm{ng} / \mathrm{ml}$ as the cutoff for AFP as instructed by the manufacturer.

\subsection{Statistical analysis}

Positive response rates of AFP and HCCR-1 were presented in terms of marginal proportions and compared by McNemar test for all patients and in each stage of clinicopathologic factors, respectively. The immunohistochemical results were analyzed by using the Pearson chi-square and Fisher's exact test. A twotailed significance level of 5\% and SAS (version 9.1) were used for all statistical analyses.

\section{Results}

\subsection{Expression of HCCR-1 in paracancerous, $L C$ and HCC tissues}

We performed the immunohistochemical analysis for HCCR-1 in 25 cases of paracancerous tissues, 14 compensating LC, 18 decompensating LC and 116 HCC tissues (Table 1). As shown in Supplementary Fig. 1, high cytoplasmic HCCR-1 staining was observed in HCCs. Although a positive staining was also exhibited in LC (compensating and decompensating) and paracancerous tissues, the HCCR-1 expression level was lower in paracancerous tissues and LC than in HCC (Supplementary Fig. 1d and Table 2). Accordingly, the HCCR-1 Allred score of 7 to 8 in HCC was $48 \%$ whereas that in paracancerous tissues, compensating, and decompensating LC was $8 \%, 7 \%$, and $22 \%$, respectively (data not shown). This suggests that HCCR-1 expression is significantly elevated in HCC compared to LC (compensating LC) and paracancerous tissues.
Table 2

Relations between HCCR-1 expression and clinicopathological characteristics of HCC and LC

\begin{tabular}{|c|c|c|c|c|}
\hline \multirow{2}{*}{$\begin{array}{l}\text { Clinical } \\
\text { characteristics }\end{array}$} & \multicolumn{2}{|l|}{$\mathrm{HCC}$} & \multicolumn{2}{|c|}{$\mathrm{LC}$} \\
\hline & + & $p$ & + & $p$ \\
\hline \multicolumn{5}{|l|}{ Sex } \\
\hline male & $79 / 100(79 \%)$ & $>0.05^{\star}$ & $14 / 32$ & $>0.05^{\star}$ \\
\hline female & $15 / 16(93.8 \%)$ & & $18 / 32$ & \\
\hline \multicolumn{5}{|l|}{ Age } \\
\hline$\geqslant 60$ & $43 / 54(79.6 \%)$ & $>0.05^{\star}$ & $13 / 32$ & $>0.05^{\star}$ \\
\hline$<60$ & $51 / 62(82.26 \%)$ & & $19 / 32$ & \\
\hline \multicolumn{5}{|l|}{ Size $(\mathrm{cm})$} \\
\hline$\geqslant 2$ & $52 / 64(81.25 \%)$ & $>0.05^{\star}$ & & \\
\hline$<2$ & $18 / 22(81.82 \%)$ & & & \\
\hline \multicolumn{5}{|l|}{ Lymph node } \\
\hline metastasis & $20 / 27(74.1 \%)$ & $>0.05^{\star}$ & & \\
\hline- & $13 / 13(100 \%)$ & & & \\
\hline+ & & & & \\
\hline \multicolumn{5}{|l|}{ Tumor grading } \\
\hline I-II & $66 / 87(75.86 \%)$ & $<0.05$ & & \\
\hline III & $28 / 29(95.6 \%)$ & & & \\
\hline
\end{tabular}

${ }^{\star}$ Fisher's exact test; $P<0.05$.

We then analyzed the relation of HCCR-1 expression to the clinicopathological characteristics of HCC and LC as shown in Table 2. The HCCR-1 level was not significantly correlated to clinicopathological characteristics such as age, sex, tumor size, and lymph node metastasis in HCC. However, the expression of HCCR1 in HCC patients with high-histological grading was significantly higher than those with low-histological grading $(P<0.05)$. Although further investigation with a larger number of tissues is needed, these results indicate that increased expression of HCCR-1 might be associated with the advanced stage of human liver carcinogenesis.

\subsection{Determination of serum cutoff values}

To determine the cutoff value of HCCR-1, we performed ROC curve analysis using 880 patients of HCC and 380 patients of $\mathrm{LC}$ or $\mathrm{CH}$ as a control. None of the patients had other malignancies or active pulmonary 
Table 3

The values of serum HCCR-1 in patients for the serum cutoff determination and representative cutoff values

3A The values of serum HCCR-1 in 380 cirrhotic or chronic hepatitis patients and $880 \mathrm{HCC}$ patients for the serum cutoff determination

\begin{tabular}{lccccc}
\hline Group & $\mathrm{N}$ & Mean & SD & Range & Median \\
\hline HCC $^{*}$ & 880 & 2436.3 & 3300.9 & $5-11401$ & 9 \\
LC or CH & 380 & 9.5 & 14.4 & $3-93$ & 6 \\
Total & 1260 & 1704.4 & 2974.7 & $3-11401$ & 8 \\
\hline${ }^{*} P<0.001$ & by Wilcoxon rank sum test.
\end{tabular}

3B Representative cutoff values with different sensitivities, specificities, and accuracies

\begin{tabular}{cccc}
\hline $\begin{array}{c}\text { Cut-off } \\
(\mathrm{ng} / \mathrm{mL})\end{array}$ & $\begin{array}{c}\text { Sensitivity } \\
(\%)\end{array}$ & $\begin{array}{c}\text { Specificity } \\
(\%)\end{array}$ & $\begin{array}{c}\text { Accuracy } \\
(\%)\end{array}$ \\
\hline 10 & 48.5 & 93.4 & 62.1 \\
9 & 59.6 & 79.2 & 65.5 \\
8 & 67.4 & 69.7 & 68.1 \\
7 & 78.3 & 54.0 & 71.0 \\
6 & 90.7 & 43.7 & 76.5 \\
\hline
\end{tabular}

disease. The mean values of serum HCCR-1 in 380 LC or $\mathrm{CH}$ patients and $880 \mathrm{HCC}$ patients were $9.5 \pm 14.4$ and $2436.3 \pm 3300.9 \mathrm{ng} / \mathrm{ml}$, respectively (Table $3 \mathrm{a}$ ). There was a statistically significant difference between the mean values of HCCR-1 in HCC patients and liver cirrhosis patients $(P<0.001)$. This result is consistent with that of the immunohistochemical stainings in Supplementary Fig. 1 showing that the HCCR-1 expression is highly elevated in HCC compared to liver cirrhosis.

ROC curve for LC and HCC patients was presented in Supplementary Fig. 2 and sensitivities, specificities, and total accuracies for five cutoff values were presented (Table 3b). We chose the value of $10 \mathrm{ng} / \mathrm{ml}$ as the cutoff for diagnostic evaluation since the specificity is over $90 \%$ at that point although the sensitivity was relatively low. Hereafter, the cutoff value for AFP and HCCR-1 will be $20 \mathrm{ng} / \mathrm{ml}$ and $10 \mathrm{ng} / \mathrm{ml}$, respectively.

\subsection{Determination of AFP and HCCR-1, alone or in combination, in normal, $\mathrm{CH}, \mathrm{LC}$ and HCC patient's sera}

This study evaluated clinical utility of AFP and HCCR-1, alone or in combination, in Chinese and Korean liver disease patients. The sensitivities of AFP and HCCR-1, alone or in combination, were increased along with the progression of liver disease (Table 4 and Supplementary Fig. 3). Combined use of AFP and HCCR-1 significantly increased the sensitivities in $\mathrm{CH}, \mathrm{LC}$ and HCC, respectively, when compared with the sensitivity of AFP or HCCR-1 alone $(P<0.001)$ (Table 4 and Supplementary Fig. 3).
The mean levels of AFP in 4 groups (N, CH, LC and HCC) showed differences between each group $(P<$ 0.0001 ), but there was no difference between normal and LC. The mean levels of HCCR-1 in 4 groups also showed differences between each group $(P<0.0001)$, but there was no difference between $\mathrm{CH}$ and LC (Supplementary Table 1).

\subsection{Diagnostic results using AFP and HCCR-1, alone or in combination related to each clinical stage, primary tumor, regional lymph nodes or distant metastasis of $\mathrm{HCC}$}

To evaluate whether AFP or HCCR-1 could be used as a biomarker for the staging of $\mathrm{HCC}$, the serum AFP or HCCR-1 level was examined in HCC patients and its association with clinicopathologic characteristics such as tumor clinical stage and TNM staging was determined. Diagnostic results of AFP compared with HCCR-1 according to the clinical stage of HCC are summarized in Table 5a. While AFP showed a significant difference between each clinical stage of HCC $(P=0.0206)$, HCCR-1 did not show any differences $(P=0.0584)$. There were no statistical differences between AFP and HCCR-1 according to each clinical stage of HCC except stage IIIB. In stage IIIB, the sensitivity of AFP was higher than that of HCCR-1 $(P<$ 0.05) (Table 5a).

Diagnostic results of HCCR-1 compared with AFP according to each primary tumor (T) of HCC are summarized in Table 5b. AFP did not show any differences in sensitivities according to each primary tumor (T) $(p=0.0564)$, and HCCR-1 also did not show any differences ( $p=0.6971$ ). There were no statistical differences between AFP and HCCR-1 according to each primary tumor (T) of HCC except T4. In T4, the sensitivity of AFP was higher than that of HCCR-1 $(P<$ 0.05) (Table 5b).

Diagnostic results of HCCR-1 compared with AFP according to each regional lymph nodes $(\mathrm{N})$ of HCC are summarized in Table 4c. AFP did not show any differences in sensitivities according to each regional lymph nodes $(\mathrm{N})$ of HCC ( $p=0.1578)$, and HCCR-1 also did not show any differences $(p=0.4855)$. There were no statistical differences between AFP and HCCR-1 according to each regional lymph nodes $(\mathrm{N})$ of HCC except NX. In NX, the sensitivity of AFP was higher than that of HCCR-1 $(P<0.05)$ (Table 5c).

Diagnostic results of HCCR-1 compared with AFP according to each distant metastasis (M) of HCC are summarized in Table 4d. AFP showed significant dif- 
Table 4

Sensitivities of AFP and HCCR-1, alone or in combination, according to the progression of liver disease

\begin{tabular}{lccccc}
\hline Tumor marker & \multicolumn{4}{c}{ Liver disease } & P value \\
\cline { 2 - 5 } & Normal & CH & LC & HCC & \\
\hline AFP & $4.2 \%(5 / 120)$ & $8.2 \%(9 / 110)$ & $26.7 \%(32 / 120)$ & $55.8 \%(164 / 294)$ & $P<0.0001$ \\
HCCR-1 & $0 \%(0 / 120)$ & $5.5 \%(6 / 110)$ & $32.5 \%(39 / 120)$ & $44.2 \%(130 / 294)$ & $P<0.0001$ \\
Combined & $4.2 \%(5 / 120)$ & $12.7 \%(14 / 110)$ & $50.0 \%(60 / 120)$ & $77.2 \%(227 / 294)$ & $P<0.0001$ \\
(AFP + HCCR-1) & & & & & \\
\hline
\end{tabular}

Table 5

Comparison of AFP with HCCR-1 related to each clinical stage, primary tumor, regional lymph nodes or distant metastasis of HCC

5A Comparison of AFP with HCCR-1 related to each clinical stage of $\mathrm{HCC}$

\begin{tabular}{cccc}
\hline Clinical stage (N) & AFP(\%) & HCCR-1(\%) & $\begin{array}{c}\text { AFP vs HCCR-1 } \\
\text { (P value) }\end{array}$ \\
\hline I (204) & 51.47 & 43.14 & $P=0.1098$ \\
II (27) & 66.67 & 51.85 & $P=0.3173$ \\
IIIA (39) & 58.97 & 43.59 & $P=0.1797$ \\
IIIB (6) & 100.00 & 33.33 & $P=0.0455$ \\
IIIC (12) & 50.00 & 25.00 & $P=0.2568$ \\
IV (6) & 100.00 & 100.00 & $P=1$ \\
\hline
\end{tabular}

5B Comparison of AFP with HCCR-1 related to each primary tumor (T) of HCC

\begin{tabular}{cccc}
\hline Primary tumor & AFP $(\%)$ & HCCR-1 $(\%)$ & AFP vs HCCR-1 \\
\hline T1 (216) & 52.78 & 43.52 & $P=0.0656$ \\
T2 (36) & 66.67 & 52.78 & $P=0.2513$ \\
T3 (36) & 55.56 & 41.67 & $P=0.2513$ \\
T4 (6) & 100.00 & 33.33 & $P=0.0455$ \\
\hline
\end{tabular}

5C Comparison of AFP with HCCR-1 in each regional lymph nodes (N) of HCC

\begin{tabular}{cccc}
\hline Regional lymph node & AFP $(\%)$ & HCCR-1 $(\%)$ & AFP vs HCCR-1 \\
\hline NX (282) & 56.03 & 45.04 & $P=0.0122$ \\
N0 (9) & 66.67 & 22.22 & $P=0.1025$ \\
N1 (3) & 0.00 & 33.33 & $P=0.3173$ \\
\hline
\end{tabular}

5D Comparison of AFP with HCCR-1 related to ach distant metastasis (M) of HCC

\begin{tabular}{cccc}
\hline Distant metastasis & AFP $(\%)$ & HCCR-1(\%) & AFP vs HCCR-1 \\
\hline MX (0) & - & - & - \\
M0 (288) & 54.86 & 43.06 & $P=0.0072$ \\
M1 (6) & 100.00 & 100.00 & $P=1$ \\
\hline
\end{tabular}

$P$-values were obtained by McNemar test.

ferences in sensitivities according to each distant metastasis $(\mathbf{M})(p=0.0359)$, and HCCR-1 also showed differences $(p=0.0070)$. The sensitivity of M1 $(100 \%)$ by AFP was significantly higher than that of M0 (54.86\%). There were no statistical differences between AFP and HCCR-1 according to each distant metastasis (M) of HCC except M0. In M0, the sensitivity of AFP was higher than that of HCCR-1 $(P<0.05)$ (Table $5 \mathrm{~d})$.

\subsection{Comparisons of AFP with HCCR-1 in HCC or HCC according to tumor size}

Comparison of diagnostic values of total serum AFP and HCCR-1 for HCC is shown in Table 5a. Of the 294 HCC patients, $164(55.8 \%)$ [95\% CI $=(50.1-61.5)$ ] and $130(44.2 \%)$ [95\% CI $=(38.5-49.9)]$ were seropositive for AFP and HCCR-1, respectively (Tables 4 and 6a). There were statistical significance between diagnostic efficiencies of AFP (55.8\%) and HCCR-1 (44.2\%) ( $P=0.0072$ by McNemar test) (Table $6 a)$. The diagnostic rate was significantly increased in combined use of AFP and HCCR-1 for HCC $(P<0.001)$ (Table 4). The positive rate in collaborative use of AFP and HCCR-1 was increased up to $77.2 \%$ (227 out of 294 HCC) (Table 4). The cases negative both for AFP and HCCR-1 were $67(22.8 \%)$. The positive rate for HCCR-1 was $48.5 \%$ (63 out of 130) in AFP-negative HCC and the positive rate for AFP was $59.1 \%$ (97 out of 164) in HCCR-1-negative HCC (Table 6a).

Many cases for AFP-negative HCC were positive for HCCR-1 and vice versa, indicating that the expression of HCCR-1 is not associated with that of AFP in many HCC. AFP and HCCR-1 were independent markers and the simultaneous use of these two markers significantly resulted in the increased sensitivity of $\mathrm{HCC}$ detection.

Among 174 large $\mathrm{HCC}(>2 \mathrm{~cm})$ excluding 120 small HCC out of total $294 \mathrm{HCC}$, the positive rates of AFP and HCCR-1 were $61.5 \%$ and $43.7 \%$, respectively (Table 6b). This shows that the diagnostic rate of AFP is higher than that of HCCR-1 in large HCC $(\geqslant 2 \mathrm{~cm})$ ( $P=0.0020$ by McNemar test), but there were no differences between AFP and HCCR-1 in HCC diagnostic results of small $\mathrm{HCC}(<2 \mathrm{~cm})$. AFP showed statistically significant high diagnostic results $(61.5 \%)$ in large HCC compared with that $(47.5 \%)$ of small HCC $(P=0.0176)$. On the contrary, there were no differences between 2 groups in case with HCCR-1 $(P=$ 0.9049).

More importantly, the diagnostic rate for small HCC was improved in the combined analysis of AFP and HCCR-1 to $70.8 \%$ although it was only $47.5 \%$ and 
$45.0 \%$ in the single analysis of AFP and HCCR-1, respectively (Table 6c). Combined analysis of AFP and HCCR-1 also markedly improved the sensitivity up to $81.6 \%$ in large HCC. Combined analysis of AFP and HCCR-1 in large HCC significantly improved the sensitivity compared with that of small HCC $(P=0.0343)$ (Table 6c) .

The combined analysis of both AFP and HCCR1 for HCC was performed and it demonstrates that the positive rate for HCC is increased up to $77.2 \%$ in HCC. In particular, the positive rate for small HCC is increased up to $70.8 \%$. This increase was significant compared to the single analysis of AFP or HCCR-1 alone. Therefore, the combined analysis of AFP and HCCR-1 improved the diagnostic rate of HCC both in large and small HCC (Table 6c). This suggests that the simultaneous detection of AFP and HCCR-1 should be effective particularly for the early diagnosis of small HCC.

As a consequence, the serological level of at least one of the two markers was elevated in a majority of HCC patients. These results suggest that the diagnostic rate can be significantly improved through the simultaneous measurement of both HCCR-1 and AFP. Therefore, HCCR-1 could be an useful tumor marker when used together with AFP for diagnosis of HCC.

\section{Discussion}

Worldwide, HCC is the 5th most common cancer and the 3rd most important cause of cancer mortality, with a 5 -year survival rate of merely $7 \%$ [24,25]. Since HCC is a disease with a very poor prognosis due to resistance to conventional chemotherapy, and mostly diagnosed at an advanced stage when most potentially curative therapies are of limited efficacy; close follow-up of patients with LC is important in order to detect HCC at an early stage. Early detection enables us to provide the patient with the most optimal therapy [26,27]. Current conventional methods for diagnosis and screening include physical examination, ultrasound imaging and serum AFP concentration measurements in high-risk patients. Despite the fact that serum AFP is still the golden standard amongst diagnostic markers for HCC, its diagnostic value is more and more questioned, due to poor sensitivity and specificity of the assay.

AFP, discovered in 1963, is an oncofetal glycoprotein that is normally produced by the fetal liver [28]. AFP levels decline gradually after birth, reaching low levels in normal adults. However, because AFP lev-
Table 6

Diagnostic evaluation of serum AFP and HCCR-1 in HCC patients

6A Diagnostic results of HCCR-1 compared with AFP in HCC patients

\begin{tabular}{lcccc}
\hline \multicolumn{5}{c}{ AFP } \\
\hline \multirow{3}{*}{ HCCR-1 } & + & 67 & 63 & $130(44.2 \%)^{*}$ \\
& - & 97 & 67 & 164 \\
& Total & $164(55.8 \%)$ & 130 & $294(100 \%)$ \\
\hline${ }^{*} P=0.0072$ by McNemar test
\end{tabular}

6B Comparison of AFP with HCCR-1 levels related to tumor sizes in $\mathrm{HCC}$

\begin{tabular}{lccc}
\hline Size & AFP $(\%)$ & HCCR-1 $(\%)$ & AFP vs HCCR-1 $(P$ value $)$ \\
\hline $2 \mathrm{~cm} \leqslant$ & 47.5 & 45.0 & $P=0.6961$ \\
$2 \mathrm{~cm}>$ & 61.5 & 43.7 & $P=0.0020$ \\
\hline$P$-values were obtained by McNemar test.
\end{tabular}

$P$-values were obtained by McNemar test.

6C Combined analysis of AFP with HCCR-1 levels related to tumor sizes in $\mathrm{HCC}$

\begin{tabular}{lccc}
\hline Size & AFP $(\%)$ & HCCR-1 $(\%)$ & AFP + HCCR-1 $(\%)^{*}$ \\
\hline $2 \mathrm{~cm} \leqslant(120)$ & 47.5 & 45.0 & 70.8 \\
$2 \mathrm{~cm}>(174)$ & 61.5 & 43.7 & 81.6 \\
\hline${ }^{*} P$-values were obtained by chi-square test $\left({ }^{*} P=0.0343\right)$.
\end{tabular}

els are usually increased in patients developing HCC, it is widely accepted as a candidate tumor marker in the detection of HCC. Previous studies have demonstrated that the sensitivity and specificity of AFP for HCC diagnosis are 41-65 and 80-94\%, respectively, with a cut-off value of $20 \mathrm{ng} / \mathrm{ml}$ [29]. This variability in the sensitivity in different studies is thought be due to several factors including study design (retrospective vs. prospective study), insufficient sample size, etiologic factors of HCC, race and the different cut-off values used for AFP. In addition, given that serum AFP levels can be increased in benign conditions such as LC, chronic hepatitis or acute hepatitis, it is difficult to determine the appropriate value at which sensitivity and specificity are maximized. Nevertheless, AFP remains the most commonly used marker in screening and surveillance programs of HCC to date.

When serum levels are markedly raised (> $400 \mathrm{ng} /$ $\mathrm{ml}$ ), as occurs in about $50 \%$ of symptomatic cases, it is particularly useful for both diagnosis of HCC and monitoring of treatment response [30,31]. However, levels of up to $400 \mathrm{ng} / \mathrm{ml}$ can be also seen in patients with chronic liver disease in the absence of HCC due to hepatocyte regeneration or exacerbation of chronic hepatitis, and there is thus a gray zone between $10 \mathrm{ng} / \mathrm{ml}$ and $400 \mathrm{ng} / \mathrm{ml}$ where the specificity of AFP for HCC is poor. For this reason, AFP is of limited use in screening cirrhotic patients for HCC, as serum AFP levels in patients with early $\mathrm{HCC}$ tend to be low while the serum 
level of AFP in cirrhotic patients without HCC may be elevated [32]. Furthermore, AFP is secreted in only about $70 \%$ of HCC, and hence both false-negative and false-positive rates are high with the use of AFP as the serological marker for detection of HCC [3].

Serological markers for hepatocellular carcinoma (HCC) are important for early diagnosis, as well as monitoring of tumour aggressiveness, treatment responsiveness, recurrence and survival. The three most common markers are total alpha-fetoprotein (AFP), Lens culinaris agglutinin-reactive AFP (AFP-L3) and protein induced by vitamin $\mathrm{K}$ absence or antagonist-II (PIVKA-II) [33]. Total AFP has the sensitivity of $60 \%$ and specificity of $90 \%$ for the detection of HCC. Increase in the percentage of AFP-L3 over the total AFP $(>10 \%)$ is very specific for small HCC. PIVKA-II is also more specific than total AFP in detecting HCC. AFP-L3 and PIVKA-II levels correlate with tumour aggressiveness and prognosis. All three markers are useful for monitoring treatment responsiveness and tumour recurrence. Since the levels of the three markers are independent of each other, combination of measurement of two or three markers will increase the sensitivity and diagnostic accuracy. Some novel markers including glypican-3 are being extensively studied.

Although the HCCR-1 oncogene was originally identified in human cervical cancer, its serum levels were found to be elevated in patients with HCC but not with cervical cancer, implying its close relationship with hepatocarcinogenesis [15]. Several laboratories have recently reported that HCCR-1 is expressed by a large proportion of HCCs, but is undetectable in normal hepatocytes and non-malignant liver disease. Furthermore, various studies demonstrated that HCCR-1 could be used as a serological test for the diagnosis of patients with HCC [18-21].

To determine the possibility of whether HCCR-1 can be used as a biomarker for HCC, $54115 \mathrm{mAb}$ and polyclonal antibody directed against HCCR-1 were generated and the serum HCCR-1 level was examined in HCC patients. Of the 294 HCC patients, $55.8 \%$ was sero-positive for AFP, and $44.2 \%$ was sero-positive for HCCR-1. The positive rate in collaborative use of AFP and HCCR-1 was increased up to $77.2 \%$. Elevated HCCR-1 levels were not associated with elevated AFP levels in most cases and the diagnostic efficiency for HCC was similar between AFP and HCCR-1. In other words, many cases for AFP-negative HCC were positive for HCCR-1 and vice versa. As a consequence, the serological level of at least one of the two markers was elevated in a majority of HCC patients.
In small HCC $(<2 \mathrm{~cm})$, the positive rate of AFP and HCCR-1 was $47.5 \%$ and $45.0 \%$, respectively. The positive rate either for AFP or HCCR-1 was increased up to $70.8 \%$ in small HCC. Therefore the combined analysis of AFP and HCCR-1 improved the diagnostic rate in small HCC.

In conclusion, our study shows that the diagnostic rate of HCC can be improved through the simultaneous measurement of both HCCR-1 and AFP. HCCR-1 could be used as a supplementary to AFP in the diagnosis of $\mathrm{HCC}$, but each of them has no satisfactory accuracy in detecting HCC or prefiguring the prognosis when used alone. Given the high heterogeneity of HCC, it is currently thought that an optimal serological test for HCC will be based on the simultaneous measurement of two or three highly specific serological markers. Combining the information from AFP and HCCR-1 increases the diagnostic rate over AFP alone in particular in small HCC and may discover the recurrence of HCC at earlier period.

\section{Conflict of interest}

No conflict of interest for any of the authors

\section{Acknowledgements}

We are grateful to the patients who participated in this project.

This work was supported by a Grant of the "KRF 2006-005-J00601”.

This work was also supported by a Grant of the "Seoul R \& BD Program" (2006).

These authors (Peng Jirun, Guoxin Zhang, and Hyun Kee Kim) contributed equally to this work.

\section{Supplementary material}

Supplementary data can be found at: http://websear ch.catholic.ac.kr/download/kimjw/supplement.doc

\section{References}

[1] H.B. El-Serag and K.L. Rudolph, Hepatocellular carcinoma: epidemiology and molecular carcinogenesis, Gastroenterology 132 (2007), 2557-2576.

[2] J. Bruix, L. Boix, M. Sala and J.M. Llovet, Focus on hepatocellular carcinoma, Cancer Cell 5 (2004), 215-219. 
[3] L. Zhou, J, Liu and F. Luo, Serum tumor markers for detection of hepatocellular carcinoma, World J Gastroenterol 12(8) (2006), 1175-1181.

[4] D.M. Parkin, F. Bray, J. Ferlay and P. Pisani, Estimating the world cancer burden: Globocan 2000, Int J Cancer 94 (2001), 153-156.

[5] M.C. Kew, Hepatocellular carcinoma. A century of progress, Clin Liver Dis 4 (2000), 257-268.

[6] M.-F. Yuen and C.-L. Lai, Serological markers of liver cancer, Best Pract Res Clin Gastroenterol 19(1) (2005), 91-99.

[7] J. Bruix, M. Sherman, J.M. Llovet et al., Clinical management of hepatocellular carcinoma. Conclusions of the Barcelona2000 EASL conference. European Association for the Study of the Liver, J Hepatol 35 (2001), 421-430.

[8] J.C. Sheu, J.L. Sung, D.S. Chen et al., Early detection of hepatocellular carcinoma by real-time ultrasonography. A prospective study, Cancer 56 (1985), 660-666.

[9] P.R. Srinivas, B.S. Kramer and S. Srivastava, Trends in biomarker research for cancer detection, Lancet Oncol 2 (2001), 698-704.

[10] D. Yao, D. Jiang, Z. Huang et al., Abnormal expressiom of hepatoma specific $\gamma$-glutamyl transferase and alteration of $\gamma$ glutamyl transferase gene methylation status in patients with hepatocellular carcinoma, Cancer 88 (2000), 761-769.

[11] M. Soresi, C. Magliarisi, P. Campagna et al., Usefulness of alpha-fetoprotein in the diagnosis of hepatocellular carcinoma, Anticancer Res 23 (2003), 1747-1753.

[12] H. Liu, P. Li, Y. Zhai et al., Diagnostic value of glypican-3 in serum and liver for primary hepatocellular carcinoma, World J Gastroenterol 16 (2010), 4410-4415.

[13] I. Bachtiar, J.M. Santoso, B. Atmanegara et al., Combination of alpha-1-acid glycoprotein and alpha-fetoprotein as an improved diagnostic tool for hepatocellular carcinoma, Clin Chim Acta 399 (2009), 97-101.

[14] N.T. Zinkin, F. Grall, K. Bhaskar et al., Serum proteomics and biomarkers in hepatocellular carcinoma and chronic liver disease, Clin Cancer Res 14 (2008), 470-477.

[15] S.K. Yoon, N.K. Lim, S.A. Ha et al., The human cervical cancer oncogene protein is a biomarker for human hepatocellular carcinoma, Cancer Res 64 (2004), 5434-5441.

[16] D. Geng, J. Chen, L. Chen et al., Effect of matrine on expression of HCCR-1 and HCCR-2 proteins in cultural human hepatocellular carcinomas cells, Chinese J Cancer Res 17(4) (2005), 265-269.

[17] J.B. Xia, Z.H. Zhang, Y.J. Tian et al., Cloning, prokaryotic expression and polyclonal antibody preparation of HCCR-1: a new biomarker for hepatocellular carcinoma, Zhonghua Gan Zang Bing Za Zhi 14(2) (2006), 101-104.

[18] Y. Yang, G.X. Zhang, R.H. Shi et al., Overexpression of
HCCR-1 in hepatocellular carcinoma cells and its clinical significance, Zhonghua Gan Zang Bing Za Zhi 15(3) (2007), 223-224.

[19] J. Ko, Y.H. Lee, S.Y. Hwang et al., Identification and differential expression of novel human cervical cancer oncogene HCCR-2 in human cancers and its involvement in p53 stabilization, Oncogene 22 (2003), 4679-4689.

[20] J. Ko, S.M. Shin, Y.M. Oh et al., Transgenic mouse model for breast cancer: induction of breast cancer in novel oncogene HCCR-2 transgenic mice, Oncogene 23 (2004), 1950-1953.

[21] G.W. Cho, S.M. Shin, H. Namkoong et al., The phosphatidylinositol 3-kinase/Akt pathway regulates the HCCR-1 oncogene expression, Gene 384 (2006), 18-26.

[22] J.N. Vauthey, G.Y. Lauwers, N.F. Esnaola et al., Simplified staging for hepatocellular carcinoma, J Clin Oncol 20(6) (2002), 1527-1536.

[23] AJCC, AJCC Cancer Staging Manual, 6th ed., Springer, New York, 2002.

[24] F.X. Bosch, J. Ribes, R. Cleries and M. Diaz, Epidemiology of hepatocellular carcinoma, Clin Liver Dis 9 (2005), 191-211.

[25] C. Verhoef, O. Visser, R.A. de Man et al., Hepatocellular carcinoma in, the Netherlands incidence, treatment and survival patterns, Eur J Cancer 40 (2004), 1530-1538.

[26] M.A. Avila, C. Berasain, B. Sangro and J. Prieto, New therapies for hepatocellular carcinoma, Oncogene 25 (2006), 38663884.

[27] P.J. Johnson, R. Williams, H. Thomas, S. Sherlock and I.M. Murray-Lyon, Induction of remission in hepatocellular carcinoma with doxorubicin, Lancet 1 (1978), 1006-1009.

[28] G.I. Abelev, S.D. Perova, N.I. Khramkova, Z.A. Postnikova and I.S. Irlin, Production of embryonal alpha-globulin by transplantable mouse hepatomas, Transplantation 1 (1963), 174-180.

[29] S. Gupta, S. Bent and J. Kohlwes, Test characteristics of alphafetoprotein for detecting hepatocellular carcinoma in patients with hepatitis C. A systematic review and critical analysis, Ann Intern Med 139 (2003), 46-50.

[30] P.J. Johnson and R. Williams, Serum alpha-fetoprotein estimations and doubling time in hepatocellular carcinoma: Influence of therapy and possible value in early detection, $J$ Nat Cancer Inst 64 (1980), 1329-1332.

[31] P.J. Johnson, The Role of Serum Alpha-fetoprotein Estimation in the Diagnosis and Management of Hepatocellular Carcinoma, in: Clinics in Liver Disease, (Vol. 5), A. Di Bisceglie, ed., WB Saunders, Philadelphia, 2001, pp. 145-159.

[32] M. Sherman, Alphafetoprotein: an obituary, J Hepatol 34 (2001), 603-605.

[33] M.F. Yuen, C.L. Lai, Serological markers of liver cancer, Best Pract Res Clin Gastroenterol 19 (2005), 91-99. 


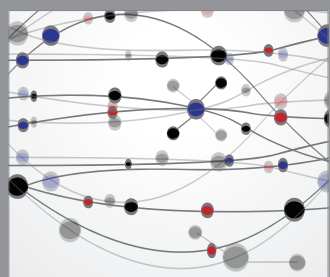

The Scientific World Journal
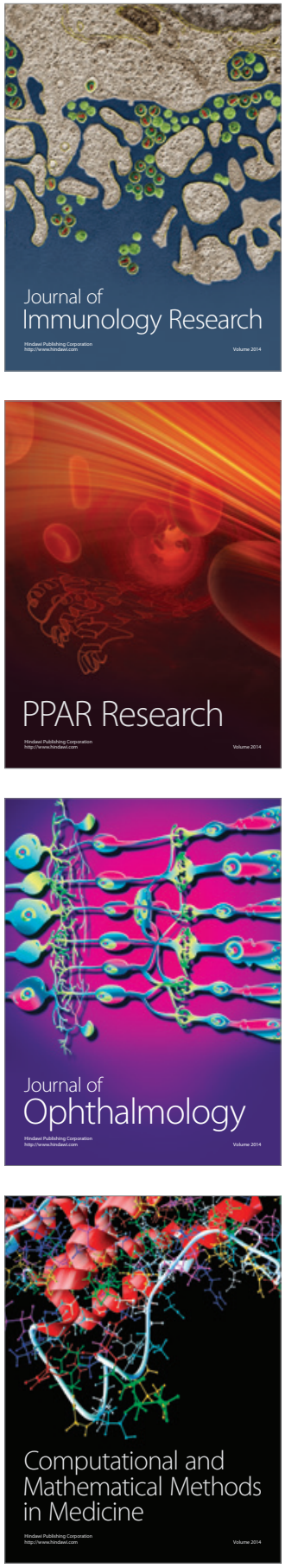

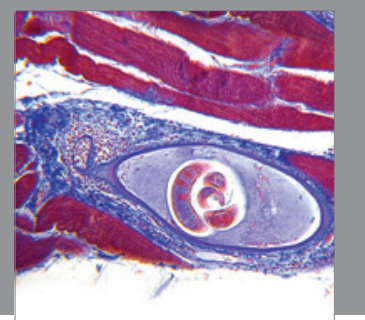

Gastroenterology

Research and Practice
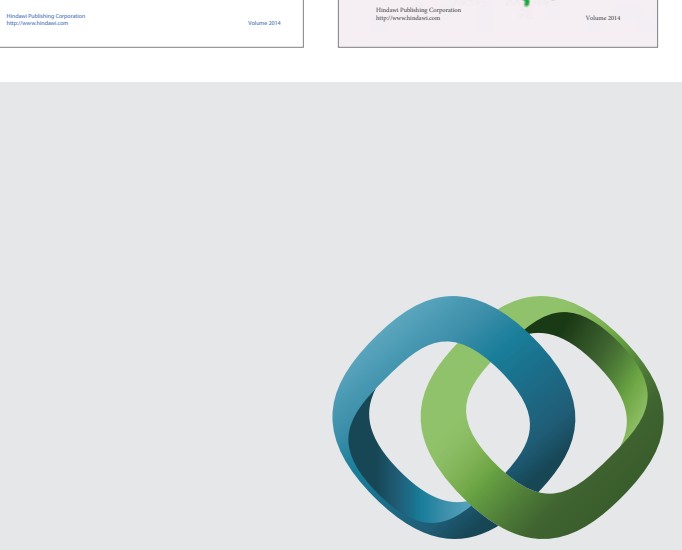

\section{Hindawi}

Submit your manuscripts at

http://www.hindawi.com
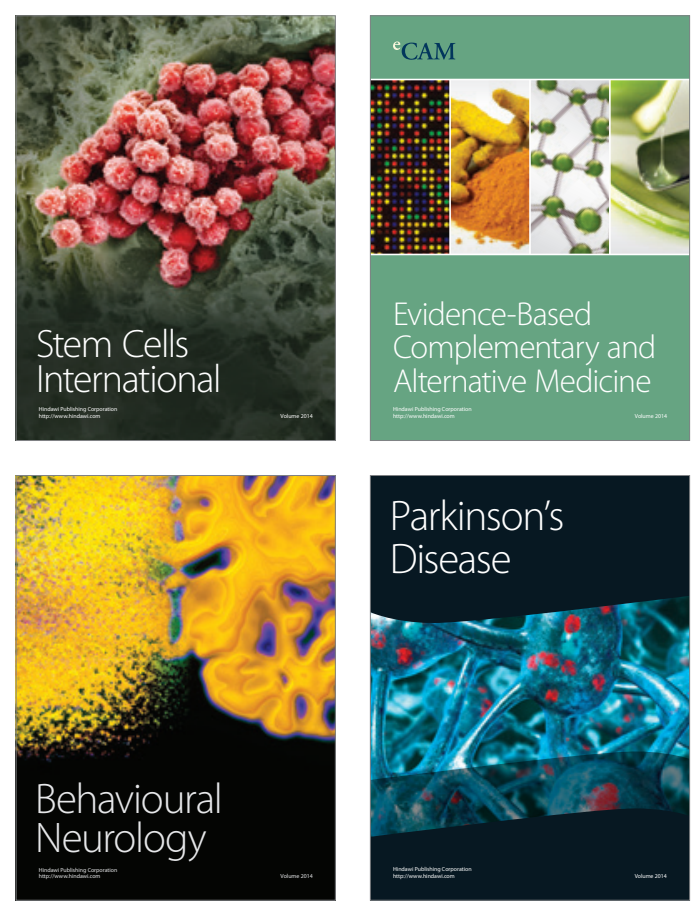

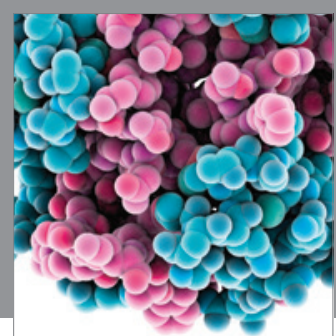

Journal of
Diabetes Research

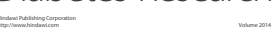

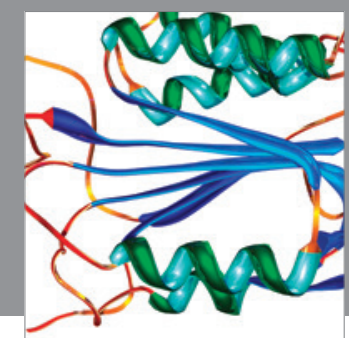

Disease Markers
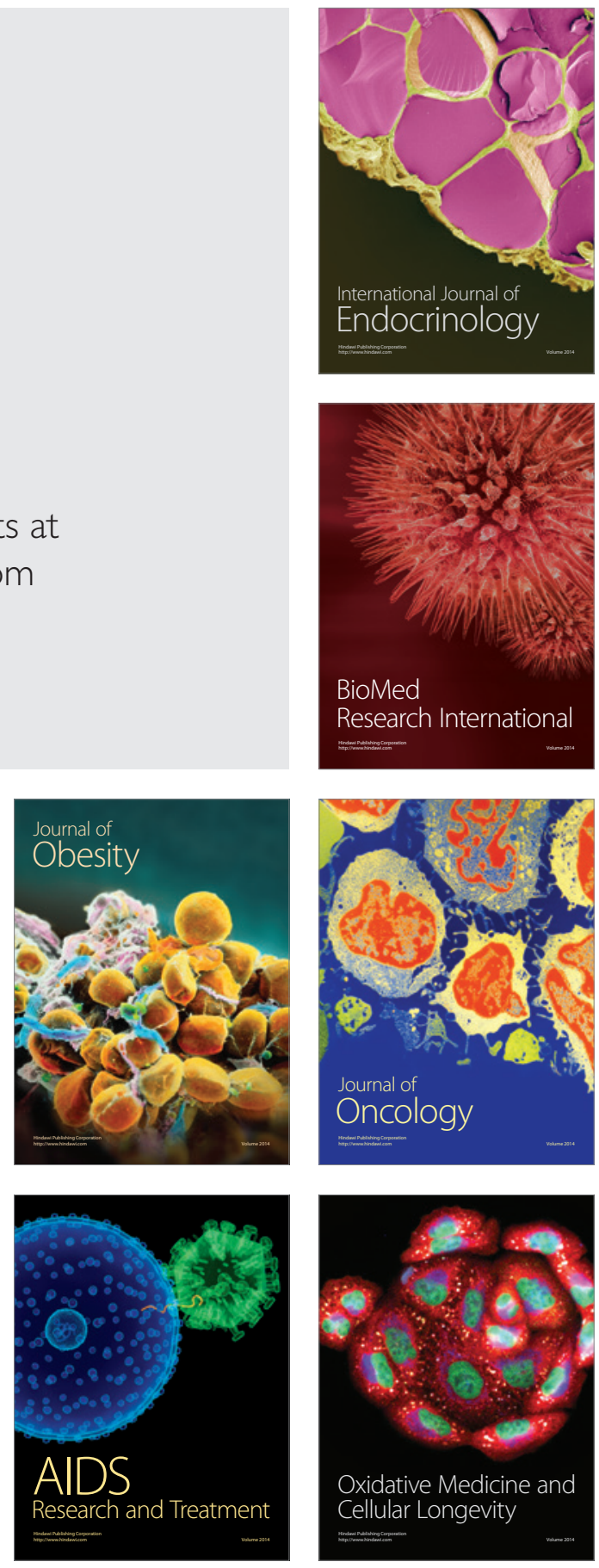